\title{
The effect of a rhythmic movement intervention on selected bio-motor skills of academy players in the Western Cape, South Africa
}

Jocelyn Solomons ${ }^{1}$, Wilbur Kraak ${ }^{1}$, Martin Kidd ${ }^{2}$ and Eileen Africa ${ }^{1}$

${ }^{1}$ Department of Sports Science, Faculty of Medicine and Health Sciences, Stellenbosch University, Stellenbosch, South Africa

${ }^{2}$ Centre for Statistical Consultation, Department of Statistics and Actuarial Sciences, Stellenbosch University, Stellenbosch, South Africa

\section{Abstract}

Rhythmic movement, also referred to as "dance", involves the execution of different motor skills as well as the integration and sequencing of actions between limbs, timing and spatial precision. The aim of this study was therefore to investigate and compare the effect of a 16-week rhythmic movement intervention on flexibility, dynamic balance, agility, power and local muscular endurance of academy rugby players in the Western Cape, according to positional group. Players $(\mathrm{N}=54)$ (age $18.66 \pm 0.81$ years; height $1.76 \pm 0.69 \mathrm{~cm}$; weight $76.77 \pm 10.69 \mathrm{~kg}$ ), were randomly divided into a treatmentcontrol $\left[\mathrm{TC}^{\mathrm{A}}\right](\mathrm{n}=28)$ and a control-treatment $\left[\mathrm{CT}^{\mathrm{B}}\right](\mathrm{n}=26)$ group. In this crossover experimental design, the interaction effect of the treatment order and the treatment time between the $T C^{A}$ and $C^{B}$ group, was determined. Results indicated a statistically significant improvement $(p<0.05)$ in agility ${ }^{2}(p=0.06)$, power ${ }^{2}(p=0.05)$, local muscular endurance $^{1}(p=0.01) \& 3(p=0.01)$ and dynamic balance $(p<0.01)$. Likewise, forwards and backs also showed statistically significant improvements $(p<0.05)$ per positional groups. Therefore, a rhythmic movement intervention has the potential to improve rugby-specific bio-motor skills and furthermore, improve positional specific skills should it be designed with positional groups in mind. Future studies should investigate, not only the effect of rhythmic movement on improving specific rugby bio-motor skills, but the potential of its application as an alternative training method during off-season (or detraining phases) or as a recovery method.

\section{Keywords}

Rhythmic movement, intervention; rugby, academy level, bio-motor skills.

\section{Introduction}

Rugby has become faster and more physically demanding because of changes in trends of match play, as well as players' physical characteristics ${ }^{1-4}$. The intermittent, 
contact nature of rugby, requires the players to have well-developed bio-motor skills, such as endurance, flexibility, balance, speed, strength and power ${ }^{5-7}$. Therefore, rugby includes various forms of fitness elements, and because of the demanding and competitive calendar, these need to get attention, both on and off the field. In terms of rugby conditioning, the common belief dictates that these fitness elements is in need of attention and should be developed through focused, isolated training blocks ${ }^{13}, 14$. However, in reality, competition structures dictate that these qualities should be developed concurrently ${ }^{15}$. For this reason, it is essential that rugby coaches and specialist coaches adapt their training methods and programmes. In turn, it will allow them to accommodate and take advantage of technical and tactical changes to the profile of the game in order to gain a competitive edge over opposing teams ${ }^{6,8-10}$.

The inclusion of other non-traditional approaches to training has become more popular within rugby conditioning. Rhythmic movement, also referred to as "dance", involves the execution of different motor skills as well as the integration and sequencing of actions between limbs, timing and spatial precision ${ }^{11}$. It requires performing movement tasks to auditory rhythmic patterns and is dependent on a large number of elements with direct and indirect effects on the physiology and physical attributes of an individual ${ }^{11,12}$. Rhythmic movement requires an athlete to demonstrate a proficient level of muscle co-ordination, muscle stamina, strength and aerobic endurance, which makes it a good non-rugby-based alternative to training bio-motor skills ${ }^{16,17}$.

The notion that rhythmic movement is beneficial as an intervention has further been explored in literature ${ }^{18-22}$. The specific and familiar example of Yoga has been used amongst many international football, rugby, cricket, and golf clubs including countries such as New Zealand, United States of America (USA) and South Africa (SA) ${ }^{23}$. Yoga is a highly structured activity that can simultaneously enhance several specific components of fitness. Similarly, to rhythmic movement, Yoga incorporates music and movement, allowing the muscles, tendons, and ligaments to move through a full range of motion. This, in turn develops and promotes balance and core strength, which is a huge advantage to athletes in their chosen sports ${ }^{23}$. Furthermore, it mimics critical aspects of athletic performance, such as flexibility, muscular strength and endurance as well as co-ordination ${ }^{23}$. A 10-week preliminary study on the impact of Yoga on specific aspects of athletic fitness amongst soccer players indicated that the group 
who practised Yoga, demonstrated improvement in both flexibility and balance ${ }^{24}$. Moreover, to emphasise how rhythmic movement contributes to other sports, can be seen in the following examples: co-ordination and isolation aspects helps with sports such as swimming and improved dynamic balance assists in sports such as gymnastics and track and field events. Furthermore, rhythmic movement teaches leaping, jumping and landing techniques for sports such as basketball, netball and rugby. Lastly, the agility and flexibility required for rhythmic movement can assist other sports in developing speed and ease of movement ${ }^{25}$.

All rugby players essentially perform some type of rhythmic movement in practise or match-play; from the duo performed between the lifters and jumpers in the line-out, the scrummaging formations to strategically timed tackles and critical displays of agility and speed to get to the try line ${ }^{7}$. The assumption is that rugby players, who also need to demonstrate a complexed interaction of the same bio-motor skills as dancers and soccer players, would benefit from a rhythmic movement intervention in the same way that soccer players experienced physical benefits. However, despite the importance of non-rugby-based approaches to training within rugby and amongst rugby clubs nowadays, recent scientific research related to this, is limited ${ }^{26}$.

Studies have shown positional differences in bio-motor skills. In a study by Jarvis et al., ${ }^{27}$ sub-elite rugby union players performed the agility ( $T$ Test and Illinois), and multistage fitness tests $(20 \mathrm{~m}, 10 \mathrm{~m}$, and $5 \mathrm{~m})$. The results indicated that backline players produced significant results $(p<0.05)$ in agility compared with forwards ${ }^{27}$. Similarly, a study on physical fitness qualities of rugby players also revealed the backs performed significantly more plyometric push-ups $(p<0.5)$ than forwards in the allocated time ${ }^{28}$. In the same study, in both the 15 - and $40 \mathrm{~m}$ sprint tests, backs were significantly faster than forwards ${ }^{10,28}$. As it relates to strength, forwards were found to possess greater absolute strength ${ }^{1,3,29}$ than backs as measured by 1RM bench press. However, no differences were found between positions in terms of 3RM squat strength and it was speculated the requirement for lower-body strength is of equal importance across all playing positions ${ }^{29}$. The aim of this study was therefore to investigate and compare the effect of a 16-week rhythmic movement intervention on flexibility, dynamic balance, agility, power and local muscular endurance of academy rugby players in the Western Cape per positional group. 


\section{Methods}

\section{Study design}

The current study was based on a crossover experimental design ${ }^{30}$. A crossover trial involves two treatments, which are administered consecutively to all the participants recruited for the study ${ }^{31}$. The main purpose served by this study design, was to provide a basis for separating treatment-effects from period-effects and to establish whether the intended outcome(s) of the intervention materialised ${ }^{31,32}$. This separation was achieved by calculating the treatment-effects separately in two sequence groups, which is done by the process of randomisation ${ }^{31}$. In this design, pre-post changes in the experimental group were directly compared to changes in the control group to indicate the effects of the intervention ${ }^{33}$. Crossover trials require a washout period to ensure that baseline data are comparable. The reversibility of a treatment effect is a prerequisite for applying a crossover design and determines the length of the washout period $^{34}$. Particularly in training studies, the washout period (anywhere between 2 and 8 weeks) is challenging, yet important, because the effect of training needs considerable time to diminish ${ }^{34}$. This testing protocol was categorised broadly under flexibility (sit- and-reach test), dynamic balance (star excursion balance test (SEBT)), agility (Illinois agility test without a ball ${ }^{1}$ and with a ball ${ }^{2}$ ), power (vertical jump test ${ }^{1}$ and seated medicine ball throw test ${ }^{2}$ ) and local muscular endurance (LME) (1-minute pushup $^{1}, 2$-minute crunch ${ }^{2}$, pull-up test ${ }^{3}$ to failure and single leg squat ${ }^{4}$ to failure). The reason for the specific testing protocol used, was because certain tests were already part of the rugby academies' testing protocol based off the national team's testing battery $^{35}$. This meant that participants were familiar with majority of the tests and the data was easily transferable to their known rugby context. Where new tests were added such as the sit and reach ${ }^{36}, \mathrm{SEBT}^{37}, 2$-minute crunch $^{38}$ and single leg squat to failure ${ }^{39}$, it was based on the most valid and reliable test which would mimic the biomotor skill that was trained during the rhythmic movement sessions.

\section{Participants}

Academy rugby players ( $N=54)$ from the Western Cape, South Africa (age $18 \pm 0.81$ years; height $1.76 \pm 0.69 \mathrm{~cm}$; weight $76.77 \pm 10.69 \mathrm{~kg}$ ), were conveniently selected to participate in this study (Table 1).

Table 1. Participant characteristics. 


\begin{tabular}{cccc}
\hline Positional groups & Age (years) & Height $(\mathrm{cm})$ & Weight $(\mathrm{kg})$ \\
\hline Forwards $(\mathrm{n}=21)$ & $18 \pm 1.72$ & $1.78 \pm 0.94$ & $79.5 \pm 14.6$ \\
Backs $(\mathrm{n}=33)$ & $18 \pm 0.20$ & $1.57 \pm 4.24$ & $71 \pm 10.6$ \\
\hline Combined $(\mathrm{N}=54)$ & $18 \pm 0.81$ & $1.76 \pm 0.69$ & $76.77 \pm 10.69$ \\
\hline
\end{tabular}

The intervention procedures were explained to the players and they were informed regarding the benefits and risks associated with the study before providing written informed consent to participate. Before commencement of the study, the players were familiarised with the testing protocol. Only players free of injury before the start of pretest 1 , were included in the study. The participants were randomly divided into a treatment-control TC $\mathrm{TC}^{\mathrm{A}}(\mathrm{n}=28)$ and control-treatment $\mathrm{CT}^{\mathrm{B}}(\mathrm{n}=26)$ group by an independent third party. Ethical approval (Ethics Project number: 7111) and insurance (Policy number: 73112118A001) was obtained from the institution.

\section{Data collection procedures}

Figure 1 presents the framework of the study. During week 1, both groups participated in pre-test 1. From weeks 2 to 9 , the treatment-control $\left(\mathrm{TC}^{\mathrm{A}}\right.$, the group who received treatment first) group was exposed to the rhythmic movement intervention, while the control-treatment $\left(\mathrm{CT}^{\mathrm{B}}\right.$, the group who received treatment in second instance) group continued their normal rugby training. In week 10, both groups completed post-test 2 . Following a washout period ${ }^{34}$ of 4 weeks between week 11 and 14, both groups participated in pre-test 2 in week 15. Thereafter, the control-CT participated in the rhythmic movement intervention, while the TC group continued their normal rugby

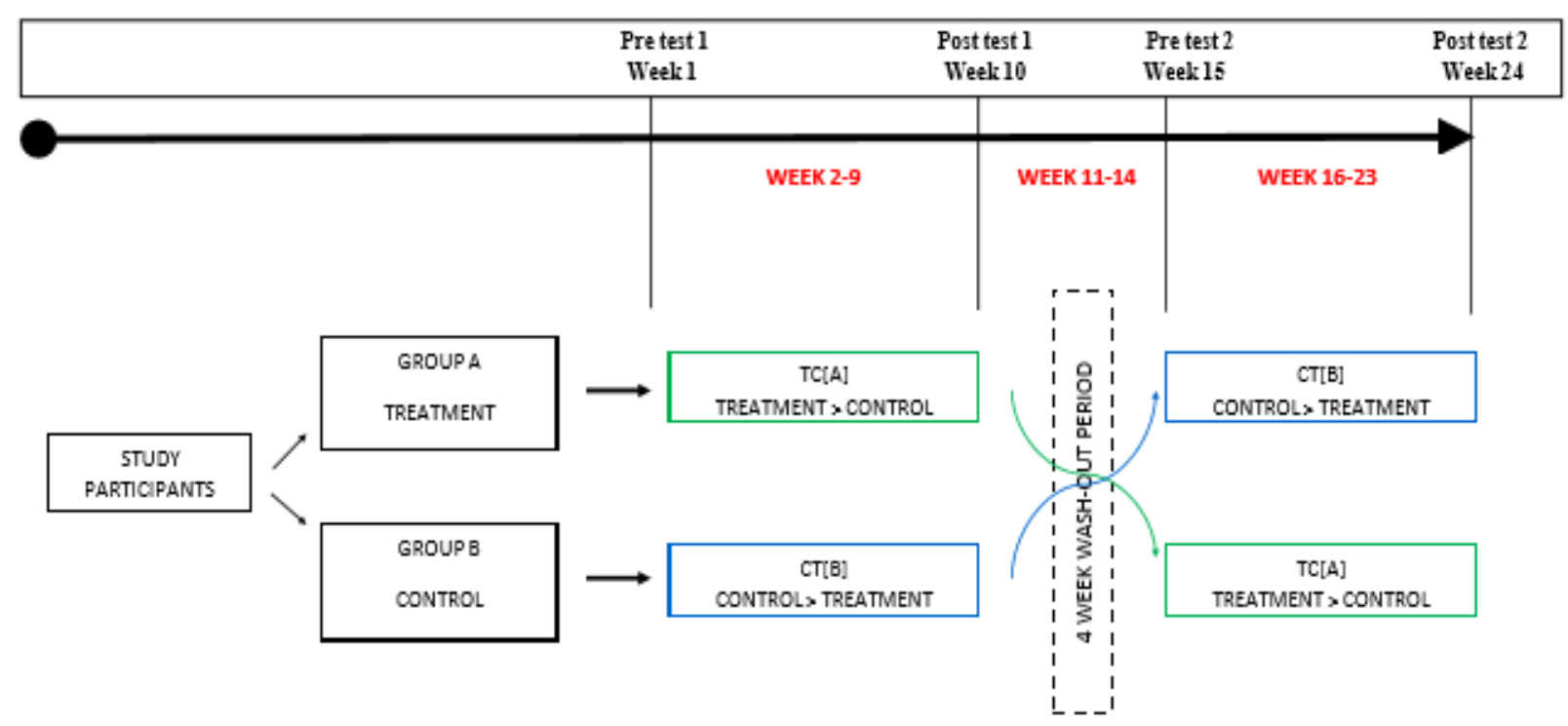


training from- and including weeks 16 to 23. Post-test 2 followed for both groups in week 24 of the intervention. The content of the intervention was exactly the same for both groups.

Figure 1. Timeline for the study.

During the pre- and post-test, all the participants were tested on various fitness elements in a field-testing order. The rhythmic movement programme was conducted and choreographed by the primary researcher who is a professional dancer and choreographer. In order to compile the intervention, the primary researcher looked at the most common movement patterns and exercises of rugby players by studying match footage. The intervention consisted of 32, 60-minute sessions over a period of 16 weeks ( $2 \times 8$ weeks). These sessions were part of their weekly planning and was not an extra session. Each session started with a 10-minute progressive aerobic endurance rhythmic movement routine as a warm-up. The warm-up was followed by 45 minutes of learning new rhythmic movement exercises and repeating it to music, which concluded with a 5-minute cool-down which involved progressive stretching.

\section{Statistical analysis}

The data was analysed using descriptive statistics (standard deviations and means). A series of one-way ANOVA with post hoc LSD t-tests were used to examine betweengroup (TCA versus $\mathrm{CT}^{\mathrm{B}}$ group) differences. Statistical significance at $5 \%(\mathrm{p}<0.05)$ was used as a guideline for determining significant results, but in keeping with recent criticism of setting significance levels, trends that were deemed important (where $p>0.05$ ) were highlighted (in cases where $p=0.06$ ).

\section{Results}

The results will be presented as follows: Table 2 and 3 depict the bio-motor skills and SEBT reach directions, respectively, which showed a statistically significant difference when comparing pre- to post- control (no treatment) and pre- to post-treatment in the different treatment groups. Table 4 includes only the bio-motor skills and Table 5 includes the SEBT directions, which showed a statistically significant difference when comparing pre- to post- control and pre- to post-treatment in the different treatment groups amongst the forwards and backs. 
Table 2: Bio-motor skills which showed a statistically significant difference, when comparing pre- to post control and pre- to post-treatment in the different treatment groups.

\begin{tabular}{|c|c|c|c|c|c|}
\hline Bio-motor skill & Treatment group & $\begin{array}{c}\text { Pre-control } \\
n=16 \\
M \pm S D\end{array}$ & $\begin{array}{c}\text { Post-control } \\
n=10 \\
M \pm S D\end{array}$ & $\begin{array}{c}\text { Pre-treatment } \\
n=15 \\
M \pm S D\end{array}$ & $\begin{array}{c}\text { Post-treatment } \\
n=13 \\
M \pm S D\end{array}$ \\
\hline \multirow[t]{2}{*}{ Agility $^{1}$} & $\mathrm{CT}$ & $17.12 \pm 2.00$ & $16.42 \pm 0.78^{*}$ & $16.52 \pm 1.58$ & $16.26 \pm 0.92$ \\
\hline & TC & $16.99 \pm 1.73$ & $16.33 \pm 0.72^{*}$ & $16.64 \pm 2.04$ & $16.26 \pm 0.87$ \\
\hline \multirow{2}{*}{ Agility $^{2}$} & $\mathrm{CT}$ & $16.72 \pm 1.30$ & $16.35 \pm 0.90$ & $16.56 \pm 0.95$ & $15.66 \pm 3.11^{*}$ \\
\hline & TC & $17.08 \pm 1.77$ & $16.30 \pm 0.63^{\star}$ & $16.50 \pm 1.30$ & $16.18 \pm 0.85$ \\
\hline \multirow{2}{*}{ Power $^{1}$} & CT & $2.81 \pm 0.13$ & $2.80 \pm 0.13$ & $2.81 \pm 0.10$ & $2.84 \pm 0.15$ \\
\hline & TC & $2.82 \pm 0.10$ & $2.76 \pm 0.09^{*}$ & $2.80 \pm .13$ & $2.78 \pm 0.13$ \\
\hline \multirow{2}{*}{ Power $^{2}$} & CT & $4.71 \pm 1.26$ & $4.60 \pm 1.07$ & $4.38 \pm 0.64$ & $4.68 \pm 0.58^{*}$ \\
\hline & TC & $4.33 \pm 0.77$ & $5.04 \pm 0.65$ & $4.78 \pm 1.27$ & $4.81 \pm 1.05$ \\
\hline \multirow{2}{*}{ LME$^{1}$} & $\mathrm{CT}$ & $48.75 \pm 16.59$ & $46.81 \pm 21.73$ & $48.76 \pm 17.63$ & $45.39 \pm 15.53$ \\
\hline & TC & $50.75 \pm 16.58$ & $43.89 \pm 10.91$ & $43.78 \pm 15.52$ & $50.64 \pm 19.44^{*}$ \\
\hline \multirow{2}{*}{$\mathrm{LME}^{3}$} & $\mathrm{CT}$ & $8.00 \pm 5.13$ & $8.58 \pm 5.34$ & $11.94 \pm 6.67$ & $10.17 \pm 9.22$ \\
\hline & TC & $11.79 \pm 5.61$ & $12.44 \pm 6.67$ & $7.19 \pm 4.50$ & $10.75 \pm 6.59^{*}$ \\
\hline \multirow{2}{*}{$\mathrm{LME}^{4}$} & CT & $32.17 \pm 20.79$ & $42.34 \pm 22.73^{*}$ & $54.32 \pm 24.34$ & $49.92 \pm 39.35$ \\
\hline & $\mathrm{TC}$ & $50.313 \pm 22.42$ & $46.09 \pm 16.46$ & $39.09 \pm 25.98$ & $43.05 \pm 14.72$ \\
\hline \multirow{2}{*}{ Flexibility } & CT & $32.56 \pm 9.83$ & $34.81 \pm 6.60$ & $322.83 \pm 5.36$ & $34.00 \pm 9.70$ \\
\hline & $\mathrm{TC}$ & $35.13 \pm 6.26$ & $35.33 \pm 8.89$ & $35.09 \pm 10.32$ & $36.43 \pm 7.56$ \\
\hline \multirow{2}{*}{$\mathrm{LME}^{2}$} & CT & $47.50 \pm 16.64$ & $48.30 \pm 16.45$ & $64.76 \pm 15.85$ & $62.10 \pm 16.43$ \\
\hline & TC & $74.65 \pm 23.32$ & $77.80 \pm 25.02$ & $47.63 \pm 14.03$ & $46.00 \pm 14.80$ \\
\hline
\end{tabular}

Note: CT - control-treatment; TC - treatment control; *significant difference between pre and post $=(p<0.05)$. 
As indicated in Table 2, there were bio-motor skills which showed a significant improvement following treatment phase. These skills included: agility ${ }^{2}$ (a trend in the CT group, $p=0.06$ ), power $^{2}$ (in the CT group, $p=0.05$ ), local muscular endurance ${ }^{1}$ (highly significant in the TC group, $\mathrm{p}=0.01$ ) and local muscular endurance ${ }^{3}$ (in the TC group, $\mathrm{p}=0.01$ ). In conclusion, no change during the control phase and a significant difference during treatment phase were observed in the above-mentioned bio-motor skills. Therefore, this is ideal results because the effect was noted during the treatment phase.

During the control phase, however, there were some bio-motor skills which showed a significant difference and these included the following: agility ${ }^{1}$ (in the CT group, $p=0.01$ \& TC group, $p=0.03$ ), agility ${ }^{2}$ (in the TC group, $p=0.02$ ), power $^{1}$ (in the TC group, $p=0.03$ ) and $\mathrm{LME}^{4}$ (in the CT group $p<0.01$ ). Due to the significant difference which was found during the time no treatment was applied, the effect cannot be attributed to the treatment.

In some cases, bio-motor skills showed no treatment effect during the control phase (pre- to post-control) nor the treatment phase (pre- to post- treatment) in either of the treatment groups (CT or TC). These bio-motor skills included: flexibility and local muscular endurance ${ }^{2}$. 
Table 3 below shows each SEBT direction. There was a significant difference seen following the treatment phase including the anterior direction (in the CT group, $p<0.01$ ), medial direction which was highly significant (in the CT group, $p<0.01$ ), anteromedial direction (in the CT group, $p<0.01$ ) and posteromedial direction (in the CT group, $\mathrm{p}<0.01$ ). It is important to note that anterior, medial, posteromedial and posterolateral directions also showed the same trends of improvement but not as significant.

During the control phase, SEBT directions which showed a significant difference during the control phase, included the medial direction (in the TC group, $p=0.01$ ), lateral direction (in the TC group, $\mathrm{p}<0.01$ ) and posterolateral direction (in the TC group, $\mathrm{p}=0.05)$.

In one instance, the posterior direction showed no treatment effect during the control phase (pre- to post-control) nor the treatment phase (pre- to post- treatment) in either of the treatment groups (CT or TC). 
Table 3: Star Excursion balance test reach directions which showed a statistically significant difference, when comparing pre- to post-control and pre- to post-treatment in the different treatment groups.

\begin{tabular}{|c|c|c|c|c|c|}
\hline Reach direction & Treatment group & $\begin{array}{c}\text { Pre-control } \\
n=16 \\
M \pm S D\end{array}$ & $\begin{array}{c}\text { Post-control } \\
n=10 \\
M \pm S D\end{array}$ & $\begin{array}{c}\text { Pre-treatment } \\
n=15 \\
M \pm S D\end{array}$ & $\begin{array}{c}\text { Post-treatment } \\
n=13 \\
M \pm S D\end{array}$ \\
\hline \multirow{2}{*}{ Anterior } & CT & $65.54 \pm 9.16$ & $67.10 \pm 7.61$ & $65.79 \pm 8.53$ & $71.05 \pm 9.57^{*}$ \\
\hline & TC & $68.36 \pm 8.79$ & $67.91 \pm 7.94$ & $65.55 \pm 9.72$ & $67.07 \pm 7.33$ \\
\hline \multirow{2}{*}{ Medial } & $\mathrm{CT}$ & $64.99 \pm 10.11$ & $64.02 \pm 11.55$ & $59.92 \pm 12.04$ & $71.18 \pm 13.25^{*}$ \\
\hline & TC & $62.16 \pm 11.67$ & $71.18 \pm 7.18^{*}$ & $64.74 \pm 12.64$ & $64.43 \pm 10.44$ \\
\hline \multirow{2}{*}{ Lateral } & $\mathrm{CT}$ & $54.80 \pm 13.08$ & $56.09 \pm 10.55$ & $58.13 \pm 11.05$ & $58.03 \pm 11.77$ \\
\hline & TC & $64.23 \pm 15.19$ & $52.18 \pm 7.10^{*}$ & $55.82 \pm 14.21$ & $55.82 \pm 8.65$ \\
\hline \multirow{2}{*}{ Anterolateral } & $\mathrm{CT}$ & $67.14 \pm 8.30$ & $65.26 \pm 8.37$ & $64.29 \pm 10.81$ & $71.74 \pm 9.52$ \\
\hline & TC & $68.52 \pm 10.19$ & $71.14 \pm 7.29^{*}$ & $66.79 \pm 8.49$ & $67.33 \pm 7.70$ \\
\hline \multirow{2}{*}{ Posterolateral } & CT & $63.82 \pm 8.55$ & $62.60 \pm 6.38$ & $63.38 \pm 6.64$ & $67.82 \pm 8.84^{*}$ \\
\hline & TC & $66.70 \pm 9.67$ & $62.50 \pm 8.77^{\star}$ & $61.68 \pm 10.00$ & $63.82 \pm 6.27$ \\
\hline \multirow{2}{*}{ Posteromedial } & $\mathrm{CT}$ & $64.89 \pm 8.10$ & $65.26 \pm 8.22$ & $64.17 \pm 8.19$ & $70.42 \pm 8.24^{*}$ \\
\hline & TC & $68.73 \pm 8.68$ & $70.55 \pm 6.01$ & $64.69 \pm 10.28$ & $66.35 \pm 7.86$ \\
\hline \multirow{2}{*}{ Anteromedial } & CT & $67.14 \pm 8.30$ & $65.26 \pm 8.37$ & $64.29 \pm 10.81$ & $71.74 \pm 9.52^{*}$ \\
\hline & $\mathrm{TC}$ & $68.52 \pm 10.19$ & $71.14 \pm 7.29$ & $66.79 \pm 8.49$ & $67.33 \pm 7.70$ \\
\hline \multirow{2}{*}{ Posterior } & $\mathrm{CT}$ & $63.91 \pm 8.46$ & $62.78 \pm 7.78$ & $65.46 \pm 7.07$ & $68.66 \pm 8.49$ \\
\hline & TC & $65.77 \pm 8.30$ & $68.23 \pm 7.24$ & $63.94 \pm 9.10$ & $64.38 \pm 6.48$ \\
\hline
\end{tabular}

Note: CT - control-treatment; TC - treatment control; * significant difference between pre and post $=(p<0.05)$ 
Table 4: Bio-motor skills which showed a statistically significant difference, when comparing pre- to post-control and pre- to post-treatment in the different treatment groups, when comparing forwards and backs.

\begin{tabular}{|c|c|c|c|c|c|c|}
\hline Bio-motor skill & Positional Group & Treatment group & $\begin{array}{c}\text { Pre-control } \\
n=16 \\
M \pm S D\end{array}$ & $\begin{array}{c}\text { Post-control } \\
n=10 \\
M \pm S D\end{array}$ & $\begin{array}{c}\text { Pre-treatment } \\
n=15 \\
M \pm S D\end{array}$ & $\begin{array}{c}\text { Post-treatment } \\
n=13 \\
M \pm S D\end{array}$ \\
\hline \multirow{4}{*}{ Agility $^{1}$} & \multirow{2}{*}{$\begin{array}{l}\text { Forwards } \\
n=21\end{array}$} & CT & $17.21 \pm 1.18$ & $16.85 \pm 0.93$ & $16.09 \pm 0.29$ & $16.82 \pm 0.79$ \\
\hline & & TC & $17.55 \pm 1.45$ & $16.52 \pm 0.81$ & $17.60 \pm 2.49$ & $16.71 \pm 0.86$ \\
\hline & \multirow{2}{*}{$\begin{array}{c}\text { Backs } \\
n=33\end{array}$} & CT & $17.06 \pm 2.40$ & $16.15 \pm 0.53^{*}$ & $16.65 \pm 1.80$ & $15.80 \pm 0.76$ \\
\hline & & TC & $16.46 \pm 1.83$ & $16.15 \pm 0.60$ & $15.93 \pm 1.24$ & $15.88 \pm 0.69$ \\
\hline \multirow{4}{*}{ Agility $^{2}$} & \multirow{2}{*}{$\begin{array}{l}\text { Forwards } \\
n=21\end{array}$} & CT & $17.62 \pm 1.49$ & $16.91 \pm 0.89$ & $16.77 \pm 1.25$ & $16.91 \pm 0.98$ \\
\hline & & TC & $17.56 \pm 1.90$ & $16.37 \pm 0.81^{*}$ & $16.90 \pm 1.08$ & $16.72 \pm 0.85$ \\
\hline & \multirow{2}{*}{$\begin{array}{l}\text { Backs } \\
n=33\end{array}$} & $\mathrm{CT}$ & $16.20 \pm 0.81$ & $16.01 \pm 0.73$ & $16.49 \pm 0.88$ & $14.68 \pm 3.82^{*}$ \\
\hline & & TC & $16.66 \pm 1.58$ & $16.24 \pm 0.43$ & $16.20 \pm 1.37$ & $15.74 \pm 0.55$ \\
\hline \multirow{4}{*}{ Power $^{1}$} & \multirow{2}{*}{$\begin{array}{l}\text { Forwards } \\
n=21\end{array}$} & $\mathrm{CT}$ & $2.83 \pm 0.15$ & $2.80 \pm 0.19^{*}$ & $2.83 \pm 0.11$ & $2.85 \pm 0.17$ \\
\hline & & TC & $2.82 \pm 0.11$ & $2.73 \pm 0.09$ & $2.82 \pm 0.14$ & $2.82 \pm 0.14$ \\
\hline & \multirow{2}{*}{$\begin{array}{l}\text { Backs } \\
n=33\end{array}$} & CT & $2.79 \pm 0.11$ & $2.80 \pm 0.07$ & $2.80 \pm 0.09$ & $2.88 \pm 0.15$ \\
\hline & & TC & $2.74 \pm 0.12$ & $2.79 \pm 0.12^{*}$ & $2.82 \pm 0.10$ & $2.81 \pm 0.04$ \\
\hline \multirow{4}{*}{ Power $^{2}$} & \multirow{2}{*}{$\begin{array}{l}\text { Forwards } \\
n=21\end{array}$} & CT & $5.45 \pm 1.22$ & $5.04 \pm 1.20$ & $4.66 \pm 0.62$ & $4.94 \pm 0.74$ \\
\hline & & TC & $4.58 \pm 0.73$ & $5.08 \pm 0.76$ & $5.22 \pm 1.33$ & $5.20 \pm 1.05$ \\
\hline & \multirow{2}{*}{$\begin{array}{l}\text { Backs } \\
n=33\end{array}$} & CT & $4.31 \pm 1.10$ & $4.32 \pm 0.90$ & $4.23 \pm 0.62$ & $4.51 \pm 0.40^{*}$ \\
\hline & & TC & $4.10 \pm 0.76$ & $4.99 \pm 0.59$ & $4.51 \pm 1.14$ & $4.50 \pm 0.97$ \\
\hline \multirow{4}{*}{$\mathrm{LME}^{1}$} & \multirow{2}{*}{$\begin{array}{c}\text { Forwards } \\
n=21\end{array}$} & $\mathrm{CT}$ & $40.00 \pm 13.15$ & $40.27 \pm 19.22$ & $44.67 \pm 10.63$ & $41.86 \pm 14.44$ \\
\hline & & $\mathrm{TC}$ & $46.92 \pm 11.24$ & $44.00 \pm 11.34$ & $37.69 \pm 14.83$ & $44.62 \pm 9.52^{*}$ \\
\hline & \multirow{2}{*}{$\begin{array}{l}\text { Backs } \\
n=33\end{array}$} & CT & $53.70 \pm 16.51$ & $51.31 \pm 22.79$ & $51.00 \pm 20.63$ & $47.64 \pm 16.46$ \\
\hline & & TC & $54.58 \pm 20.41$ & $43.75 \pm 12.09$ & $48.47 \pm 15.01$ & $55.87 \pm 24.26$ \\
\hline
\end{tabular}




\begin{tabular}{|c|c|c|c|c|c|c|}
\hline Bio-motor skill & Positional Group & Treatment group & $\begin{array}{c}\text { Pre-control } \\
n=16 \\
M \pm S D\end{array}$ & $\begin{array}{c}\text { Post-control } \\
n=10 \\
M \pm S D\end{array}$ & $\begin{array}{c}\text { Pre-treatment } \\
n=15 \\
M \pm S D\end{array}$ & $\begin{array}{c}\text { Post-treatment } \\
n=13 \\
M \pm S D\end{array}$ \\
\hline \multirow{4}{*}{$\mathrm{LME}^{2}$} & \multirow{2}{*}{$\begin{array}{c}\text { Forwards } \\
n=21\end{array}$} & CT & $40.00 \pm 13.22$ & $46.18 \pm 7.96$ & $60.00 \pm 5.48$ & $58.13 \pm 17.55$ \\
\hline & & $\mathrm{TC}$ & $66.00 \pm 19.74$ & $76.00 \pm 24.44$ & $44.00 \pm 12.71$ & $41.38 \pm 14.09$ \\
\hline & \multirow{2}{*}{$\begin{array}{c}\text { Backs } \\
n=33\end{array}$} & $\mathrm{CT}$ & $51.74 \pm 17.12$ & $49.75 \pm 20.52^{*}$ & $67.36 \pm 19.13$ & $64.75 \pm 15.85$ \\
\hline & & $\mathrm{TC}$ & $83.31 \pm 24.11$ & $79.60 \pm 28.34$ & $50.68 \pm 14.69$ & $49.75 \pm 14.72$ \\
\hline \multirow{4}{*}{$\mathrm{LME}^{3}$} & \multirow{2}{*}{$\begin{array}{c}\text { Forwards } \\
n=21\end{array}$} & CT & $5.15 \pm 4.76$ & $6.36 \pm 4.12$ & $7.33 \pm 1.86$ & $5.86 \pm 6.72$ \\
\hline & & $\mathrm{TC}$ & $11.25 \pm 5.94$ & $13.40 \pm 8.71$ & $5.63 \pm 4.60$ & $10.00 \pm 7.22^{*}$ \\
\hline & \multirow{2}{*}{$\begin{array}{c}\text { Backs } \\
n=33\end{array}$} & CT & $9.68 \pm 4.66$ & $10.20 \pm 5.67$ & $14.45 \pm 7.05$ & $12.91 \pm 9.80$ \\
\hline & & $\mathrm{TC}$ & $12.33 \pm 5.47$ & $11.25 \pm 3.77$ & $8.68 \pm 5.08$ & $11.40 \pm 6.17$ \\
\hline \multirow{4}{*}{$\mathrm{LME}^{4}$} & \multirow{2}{*}{$\begin{array}{c}\text { Forwards } \\
n=21\end{array}$} & $\mathrm{CT}$ & $37.09 \pm 21.06$ & $47.09 \pm 25.94$ & $60.36 \pm 26.19$ & $53.77 \pm 48.53$ \\
\hline & & $\mathrm{TC}$ & $47.33 \pm 21.27$ & $43.00 \pm 14.30$ & $39.38 \pm 21.62$ & $43.79 \pm 14.74$ \\
\hline & \multirow{2}{*}{$\begin{array}{c}\text { Backs } \\
n=33\end{array}$} & CT & $37.09 \pm 21.06$ & $47.09 \pm 25.94^{*}$ & $60.36 \pm 26.19$ & $53.77 \pm 48.53$ \\
\hline & & $\mathrm{TC}$ & $47.33 \pm 21.27$ & $43.00 \pm 14.30$ & $39.38 \pm 21.62$ & $43.79 \pm 14.74$ \\
\hline
\end{tabular}

Note: CT - control-treatment; TC - treatment control; * significant difference between pre and post $=(p<0.05)$. 
Table 4 above shows the bio-motor skills which showed a statistically significant difference when comparing pre- to post-control and pre- to post-treatment in the different groups, when comparing the forwards and backs. Amongst the forwards, the bio-motor skills which showed a significant difference (an improvement) at the time when treatment was applied, included the following: local muscular endurance ${ }^{1}$ (TC group, $p=0.03$ ) and local muscular endurance ${ }^{3}$ (TC group, $p=0.02$ ). Amongst the backs, bio-motor skills which showed a significant difference (an improvement) at the time when treatment was applied, included agility ${ }^{2}$ (CT group, $p<0.01$ ), power $^{2}$ (CT group, $p=0.04)$. Moreover, particularly in agility ${ }^{2}$ and power ${ }^{2}$ bio-motor skills, although a significant effect was seen in one treatment group only, the same non-significant trend was seen in the other (TC) treatment group.

In other cases, the effect amongst backs could not be attributed to the treatment because significant differences were seen during the control phase. These skills included: agility ${ }^{1}$ (in the CT group, $p<0.01$ ), local muscular endurance ${ }^{4}$ (in the CT group, $p=0.01$ ). Although there were no significant differences observed during the treatment time, a significant decrease in these bio-motor skills may be attributed to the fact that no treatment was presented. 
Table 5: SEBT reach directions which showed a statistically significant difference $(p=<0.05)$, when comparing pre- to post-control (no treatment) and pre- to post-treatment $(\mathrm{M} \pm \mathrm{SD}$ ) in the different treatment groups (TC and $\mathrm{CT}$ ), when comparing forwards and backs.

\begin{tabular}{|c|c|c|c|c|c|c|}
\hline Bio-motor skill & Positional Group & Group & $\begin{array}{c}\text { Pre-control } \\
n=16\end{array}$ & $\begin{array}{c}\text { Post-control } \\
n=10\end{array}$ & $\begin{array}{c}\text { Pre-treatment } \\
n=15 \\
\end{array}$ & $\begin{array}{c}\text { Post-treatment } \\
n=13\end{array}$ \\
\hline \multirow{4}{*}{ Anterior } & \multirow{2}{*}{$\begin{array}{l}\text { Forwards } \\
\qquad n=21\end{array}$} & CT & $65.73 \pm 8.56$ & $68.63 \pm 7.65$ & $62.00 \pm 8.00$ & $74.50 \pm 8.61^{*}$ \\
\hline & & TC & $70.36 \pm 8.69$ & $68.25 \pm 5.43$ & $65.59 \pm 11.43$ & $69.77 \pm 8.20^{\star}$ \\
\hline & \multirow{2}{*}{$\begin{array}{l}\text { Backs } \\
n=33\end{array}$} & $\mathrm{TC}$ & $43.79 \pm 14.74$ & $23.52 \pm 17.53$ & $35.00 \pm 14.25$ & $43.25 \pm 16.15$ \\
\hline & & CT & $44.27 \pm 19.77$ & $53.29 \pm 23.59$ & $47.86 \pm 17.84$ & $38.68 \pm 31.13$ \\
\hline \multirow{4}{*}{ Posterior } & \multirow{2}{*}{$\begin{array}{c}\text { Forwards } \\
n=21\end{array}$} & CT & $62.80 \pm 9.35$ & $61.21 \pm 8.60$ & $65.50 \pm 4.55$ & $74.21 \pm 10.63^{*}$ \\
\hline & & $\mathrm{TC}$ & $69.23 \pm 6.41$ & $69.83 \pm 5.56$ & $64.72 \pm 8.34$ & $66.12 \pm 7.23$ \\
\hline & \multirow{2}{*}{$\begin{array}{l}\text { Backs } \\
n=33\end{array}$} & $\mathrm{TC}$ & $64.66 \pm 7.82$ & $63.88 \pm 7.05$ & $65.44 \pm 7.85$ & $65.42 \pm 4.74$ \\
\hline & & CT & $62.32 \pm 8.67$ & $66.30 \pm 8.78$ & $62.95 \pm 9.64$ & $63.06 \pm 5.60$ \\
\hline \multirow{4}{*}{ Medial } & \multirow{2}{*}{$\begin{array}{l}\text { Forwards } \\
\qquad n=21\end{array}$} & CT & $64.63 \pm 9.69$ & $66.46 \pm 11.84$ & $58.83 \pm 14.74$ & $75.93 \pm 15.62^{\star}$ \\
\hline & & $\mathrm{TC}$ & $65.18 \pm 11.20$ & $71.50 \pm 7.59$ & $65.91 \pm 13.96$ & $67.08 \pm 9.17$ \\
\hline & \multirow{2}{*}{$\begin{array}{l}\text { Backs } \\
n=33\end{array}$} & CT & $65.23 \pm 10.49$ & $62.29 \pm 11.19$ & $60.28 \pm 11.48$ & $68.42 \pm 11.09^{*}$ \\
\hline & & $\mathrm{TC}$ & $59.14 \pm 11.59$ & $70.80 \pm 7.04^{*}$ & $63.93 \pm 11.80$ & $62.41 \pm 11.03$ \\
\hline \multirow{4}{*}{ Lateral } & \multirow{2}{*}{$\begin{array}{c}\text { Forwards } \\
n=21\end{array}$} & $\mathrm{CT}$ & $52.17 \pm 15.69$ & $55.50 \pm 10.57$ & $57.50 \pm 12.72$ & $59.93 \pm 12.24$ \\
\hline & & TC & $68.36 \pm 15.39$ & $56.33 \pm 5.18^{*}$ & $54.88 \pm 14.83$ & $56.00 \pm 9.27$ \\
\hline & \multirow{2}{*}{$\begin{array}{l}\text { Backs } \\
n=33\end{array}$} & CT & $56.59 \pm 10.78$ & $56.50 \pm 10.68$ & $58.33 \pm 10.83$ & $56.92 \pm 11.61$ \\
\hline & & $\mathrm{TC}$ & $60.09 \pm 14.13$ & $47.20 \pm 5.85^{\star}$ & $56.34 \pm 13.89$ & $55.68 \pm 8.28$ \\
\hline \multirow{4}{*}{ Anterolateral } & \multirow{2}{*}{$\begin{array}{l}\text { Forwards } \\
\qquad n=21\end{array}$} & CT & $62.30 \pm 10.96$ & $62.88 \pm 8.79$ & $62.00 \pm 8.25$ & $67.86 \pm 11.18$ \\
\hline & & TC & $71.82 \pm 9.85$ & $63.33 \pm 5.55^{*}$ & $64.50 \pm 13.35$ & $64.81 \pm 7.21$ \\
\hline & \multirow{2}{*}{$\begin{array}{l}\text { Backs } \\
n=33\end{array}$} & CT & $63.55 \pm 9.52$ & $64.24 \pm 7.70$ & $64.00 \pm 8.76$ & $65.04 \pm 8.70$ \\
\hline & & TC & $64.00 \pm 10.77$ & $60.40 \pm 12.27$ & $65.36 \pm 10.43$ & $63.88 \pm 6.14$ \\
\hline
\end{tabular}




\begin{tabular}{|c|c|c|c|c|c|c|}
\hline Bio-motor skill & Positional Group & Group & $\begin{array}{c}\text { Pre-control } \\
n=16\end{array}$ & $\begin{array}{c}\text { Post-control } \\
n=10\end{array}$ & $\begin{array}{c}\text { Pre-treatment } \\
n=15\end{array}$ & $\begin{array}{c}\text { Post-treatment } \\
n=13\end{array}$ \\
\hline \multirow{4}{*}{ Anteromedial } & \multirow{2}{*}{$\begin{array}{c}\text { Forwards } \\
n=21\end{array}$} & $\mathrm{CT}$ & $67.13 \pm 7.81$ & $65.75 \pm 9.04$ & $60.00 \pm 10.45$ & $74.50 \pm 10.32^{*}$ \\
\hline & & TC & $71.05 \pm 8.99$ & $70.00 \pm 6.21$ & $67.81 \pm 8.83$ & $68.81 \pm 7.54$ \\
\hline & \multirow{2}{*}{$\begin{array}{c}\text { Backs } \\
n=33\end{array}$} & CT & $67.14 \pm 8.71$ & $64.91 \pm 7.97$ & $65.77 \pm 10.83$ & $70.13 \pm 8.84$ \\
\hline & & TC & $66.00 \pm 10.88$ & $72.50 \pm 8.54^{*}$ & $65.93 \pm 8.42$ & $66.21 \pm 7.74$ \\
\hline \multirow{4}{*}{ Posterolateral } & \multirow{2}{*}{$\begin{array}{c}\text { Forwards } \\
n=21\end{array}$} & CT & $62.97 \pm 9.0$ & $63.92 \pm 5.26$ & $62.17 \pm 5.19$ & $69.79 \pm 10.67$ \\
\hline & & TC & $69.36 \pm 7.79$ & $61.92 \pm 9.82^{*}$ & $60.69 \pm 9.28$ & $65.73 \pm 6.30^{\star}$ \\
\hline & \multirow{2}{*}{$\begin{array}{c}\text { Backs } \\
n=33\end{array}$} & CT & $64.41 \pm 8.29$ & $61.62 \pm 6.98$ & $63.78 \pm 7.14$ & $66.67 \pm 7.59$ \\
\hline & & TC & $64.05 \pm 10.77$ & $63.20 \pm 7.79$ & $62.48 \pm 10.65$ & $62.35 \pm 5.91$ \\
\hline \multirow{4}{*}{ Posteromedial } & \multirow{2}{*}{$\begin{array}{c}\text { Forwards } \\
n=21\end{array}$} & $\mathrm{CT}$ & $65.00 \pm 8.73$ & $65.46 \pm 8.81$ & $63.50 \pm 6.95$ & $73.14 \pm 10.09^{*}$ \\
\hline & & TC & $71.82 \pm 7.33$ & $69.92 \pm 6.29$ & $63.88 \pm 10.95$ & $67.96 \pm 7.49^{*}$ \\
\hline & \multirow{2}{*}{$\begin{array}{c}\text { Backs } \\
n=33\end{array}$} & CT & $64.82 \pm 7.75$ & $65.12 \pm 7.91$ & $64.39 \pm 8.73$ & $68.83 \pm 6.66^{*}$ \\
\hline & & TC & $65.64 \pm 8.97$ & $71.30 \pm 5.89$ & $64.91 \pm 9.87$ & $65.12 \pm 8.02$ \\
\hline
\end{tabular}

Note: CT - control-treatment; TC - treatment control; ${ }^{*}$ significant difference between pre and post $=(p<0.05)$ 
Table 5 shows significant differences for forwards in the SEBT anterior direction (TC group, $p=0.01$ and CT group, $p<0.01$ ), posterior direction (in the CT group, $p=0.01$ ), medial direction (CT group, $p<0.01$ ), anteromedial direction (CT group, $p<0.01$ ), posterolateral direction (TC group, $\mathrm{p}=0.01$ ) and posteromedial direction (CT group, $p=0.03)$ and the $T C$ group $(p=0.06)$. During the control phase, a significant decrease was seen including the following SEBT directions: lateral (in the TC group, $p=0.01$ ), anterolateral (in the TC group, $p=0.03$ ) and posterolateral (in the TC group, $p=0.01$ ).

Amongst backs during the treatment phase, significant differences were seen in the medial direction (CT group, $\mathrm{p}=0.01$ ) and posteromedial direction (CT group, $\mathrm{p}<0.01$ ). During the control phase, a significant increase was noted in the medial direction (in the TC group, $\mathrm{p}=0.01$ ) and a trend in the anteromedial direction (in the TC group, $p=0.06)$. Moreover, significant decreases were noted during the control phase in the medial (in the TC group, $\mathrm{p}=0.01$ ) and anteromedial direction (a trend in the TC group, $\mathrm{p}=0.06$ ). In summary, flexibility was the only bio-motor skill, which did not show a significant difference amongst the forwards and/or backs when the position and treatment time was considered. However, it must be noted that flexibility did show a significant difference in the combined treatment groups amongst forwards $(p=0.05)$; the TC group showed to benefit more than the CT group in this case. Furthermore, in instances where there was no significant difference seen in the treatment groups, some skills in fact showed a significant treatment effect for the treatment groups combined. These skills included: agility $^{2}$ and SEBT anterior, posterior, medial, anteromedial, posterolateral, and posteromedial direction.

\section{Discussion}

The study reflected 3 main outcomes: (1) the intervention was effective in improving certain bio-motor skills at the time when treatment was implemented, (2) where improvements did occur, it could not be (solely) attributed to the intervention; and (3) there is potential for positional group improvement in performance of selected rugby bio-motor skills, if the intervention is designed with positional groups in mind.

The aim of this study was to investigate and compare the effect of a 16-week rhythmic movement intervention on flexibility, dynamic balance, agility, power and local muscular endurance of academy rugby players in the Western Cape per positional group. The major findings of the study conclude that statistically significant differences 
were found in agility, power, local muscular endurance and certain dynamic balance bio-motor skills and that significant improvements of certain bio-motor skills were also noted per positional group (forwards and backs). According to the researcher's knowledge, this is the first study to investigate the effect of a rhythmic movement intervention on selected bio-motor skills among academy rugby players.

The improvement and thus, positive performance of bio-motor skills evident amongst forwards and backs, corresponds with findings from Barr et al. ${ }^{40}$ who notes that lower body power can assist sprinting ability by improving general maximal strength. Considering local muscular endurance, a study by Kloubec et al. ${ }^{41}$ revealed improvements in local muscular endurance following pilates exercises for two 60minute sessions per week over a 12-week period. From this, the primary researcher of the current study can presume that a higher intensity, multi-faceted rhythmic movement intervention over 16 weeks (two, 8-week periods), also has potential to improve muscular endurance. In agreement with Kloubec et al. ${ }^{41}$, the findings of the current study indeed revealed a statistically significant improvement in local muscular endurance ${ }^{1 \& 3}$ overall and specifically amongst forwards.

According to Duthie et al. ${ }^{42}$, there are clear differences in the physiological and anthropometric traits of forwards and backs. Forwards and backs in the current study, showed different amounts of improvements across the bio-motor skills, which may allude to positional differences during match play. The current study found a statistically significant difference amongst backs in terms of agility ${ }^{2}$, power ${ }^{2}$, medial and posteromedial directions of the SEBT in one treatment group only (the CT group). Forwards improved in local muscular endurance ${ }^{1 \& 3}$, as well as SEBT directions anterior, posterior, anteromedial, posterolateral and posteromedial. In agreement with Duthie et al. ${ }^{42}$ and Durandt ${ }^{43}$ positional differences certainly do exist between these two groups and they need to be trained accordingly. The difference in bio-motor skill performance between forwards and backs can be accredited to the different positional roles they fulfil in a game. Compared to backs, forwards experience sustained higher contact loads per match because of activities, such as tackles, rucks and mauls ${ }^{40,42}$. They require different physical conditioning because of the number of impacts in the game $^{40}$. This could also be attributed to the difference in height and weight as revealed by the player characteristics of the current study. As noted by Quarrie et al. ${ }^{44}$, it is evident that each position has specific functional roles during match play, as well as 
bio-motor skill requirements and therefore, requires specific fitness and conditioning components to suite these various requirements. Due to this being a novel study, the primary researcher had no previous knowledge or insights as to which rhythmic movements would work best to illicit change or improve performance in bio-motor skills, what type of music would be easy for the participants to perform the movements to and more importantly, how long it would take for each specific bio-motor skill to show improvement. Where differences did occur in certain bio-motor skills, these were statistically significant not only amongst the entire group of participants, but also when considering per positional group differences. Overall, the lack in performance improvement and often minimal change in performance regarding bio-motor skills, may allude to the treatment on its own being inefficient in its ability to target improvement in specific bio-motor skills over the short period of time. Additionally, the improvement subsequent to no treatment may indicate that the rugby academies' conditioning programme had an effect on the bio-motor skills, while the rhythmic movement treatment was not implemented. The results from this study highlighted the importance for further research on the effect of rhythmic movement for rugby specific bio-motor skills.

\section{Practical application}

In terms of the practical application of the current study, rhythmic movement is an easy and enjoyable alternative method for training bio-motor skills. Coaches may use the various parts of the rhythmic movement sessions and adapt it to a specific training focus.

The warm-up of a rhythmic movement session allows for a dynamic, progressive preparation to any physical training session by including mobility work to music. It is useful to include a 15 to 20 minute rhythmic movement session before starting a rugbyspecific session whether it is prior to a contact field session or gym-based strength training. A warm-up rhythmic movement session can thus be seen as preparation for any other session to follow. The benefits ${ }^{43}$ of music in sport and exercise have reflected benefits of music in terms of mood, affect and cognition (psychological), psychophysical effects (perception of physical effort), psycho-physiological (heart and respiration rate), and ergogenic effects. 
The body of a rhythmic movement session can be utilised to focus on specific biomotor skills training. A focus on speed and power ${ }^{44}$, would mean that coaches can spend 30 minutes on movements related to generating speed and power. Movements may include a variety of quick feet actions to focus on technique in order to produce foot speed and rhythm (using steps from salsa dancing with the inclusion of agility ladders) or, lower body power moves (using vertical jumps as in the case of Ballet). Studies have reported the existence and importance of rhythm in sport skills. CôtéLaurence ${ }^{45}$ reports that sense of rhythm applies to ball games, which helps develop attitudes of calmness and fluency for performers.

Furthermore, local muscular endurance showed an improvement in the study which showed that the use of rhythmic movements involved in the "cha cha slide", may be used in a session to improve local muscular endurance. As in the intervention during the study, this specific well-known line-dance, can be done in plank position to target whole body endurance or, standing upright but at a quicker pace to work on agility, speed and decision making. The cool down of a rhythmic movement is just as important as the warm-up and session itself. Flexibility was the only bio-motor skill which did not show a significant improvement. Various other forms of stretching approaches (such as the inclusion of Yoga) may be more beneficial to players' overall mobility and flexibility. Particularly as it relates to neck, lower back and hamstring flexibility for players involved in the scrum, utilising rhythmic movements for- and in this position, may contribute to better technique in the scrum. The use of various mobility and flexibility rhythmic movements can help coaches to train technical elements with a different method. A time for players to recover from a demanding session, should not be taken lightly. Often the next conditioning session is influenced by the preceding session. A rhythmic movement session can therefore assist in mental and physical recovery from field sessions as well as matches during the week/ends.

Rhythmic movement can be adapted in various ways in terms of music and movement to accommodate not only the rugby players, but also rugby-orientated movements. Finally, a rhythmic movement intervention can be used throughout the season with adaptations to intensity according to the demands of the training phase. For this reason, multiple bio-motor skills can be trained simultaneously, which is advantageous to a demanding rugby-training schedule. In other words, rhythmic movement can be 
used as a tool to warm-up, as a conditioning method to improve specific bio-motor skills, technical skills or, when required, as a recovery method for players.

\section{Conclusion}

The study concluded that there were significant improvements in certain bio-motor skills over the short period of time. More specifically, major findings of the study revealed statistically significant improvements from pre- to post-treatment in agility, power, dynamic balance and local muscular endurance. Additionally, when considering forwards and backs, further improvements were seen in certain -bio-motor skills per positional group.

Limitations included the continuation of normal training, individual schedules and rugby conditioning programmes for the academy players and the content of the sessions not being designed to accommodate positional specific demands and biomotor skills. Future research first needs to determine which types of rhythmic movements will most effectively illicit improvements on rugby specific bio-motor skills. Thereafter, a rhythmic movement intervention based on the specific rugby related rhythmic movements should be compiled and implemented. Studies should investigate, not only the effect of rhythmic movement on improving specific rugby biomotor skills, but also its application as an alternative training method during off-season (or detraining phases) or as a recovery method.

\section{Acknowledgements}

The authors would like to thank the South African National Research Foundation for the financial assistance towards the research and the players who participated in the study.

\section{Declaration of conflicting interests}

The author(s) declared no potential conflicts of interest with respect to the research, authorship, and/or publication of this article.

\section{Funding}

This work was supported by the South African National Research Foundation. (SFH180531337839) by financial assistance towards the research. 


\section{References}

1. Lombard WP, Durandt JJ, Masimla H, Green M, Lambert MI., Changes in body size and physical characteristics of South African under-20 rugby union players over a 13-year period, Journal of Strength and Conditioning Research, 2015, 29(4), 980-8.

2. Owen SM, Venter RE, Du Toit S, Kraak WJ., Acceleratory match-play demands of a Super Rugby team over a competitive season, Journal of Sports Sciences, 2015, 33(19), 2061-9.

3. Jones B, Weaving D, Tee J, Darrall-Jones J, Weakley J, Phibbs P, Read D, Roe G, Hendricks S, Till K., Bigger, stronger, faster, fitter: the differences in physical qualities of school and academy rugby union players, Journal of Sports Sciences, 2018, 36(21), 2399-404.

4. Read DB, Jones B, Phibbs PJ, Roe GA, Darrall-Jones J, Weakley JJ, Till K., The physical characteristics of match-play in English schoolboy and academy rugby union, Journal of Sports Sciences, 2018, 36(6), 645-50.

5. Argus CK, Gill N, Keogh J, Hopkins WG, Beaven CM., Effects of a short-term pre-season training programme on the body composition and anaerobic performance of professional rugby union players, Journal of Sports Sciences, 2010, 28(6), 679-86.

6. Austin D, Gabbett T, Jenkins D., The physical demands of Super 14 rugby union, Journal of Science and Medicine in Sport, 2011, 14(3), 259-63.

7. Gabbett TJ, Jenkins DG, Abernethy B., Physical demands of professional rugby league training and competition using microtechnology, Journal of Science and Medicine in Sport, 2012, 15(1), 80-6.

8. Hartwig TB, Naughton G, Searl J., Motion analyses of adolescent rugby union players: a comparison of training and game demands, Journal of Strength and Conditioning Research, 2011, 25(4), 966-72.

9. Handcock $P$, Cassidy $T$., Reflective practice for rugby union strength and conditioning coaches, Strength and Conditioning Journal, 2014, 36(1), 41-5.

10. Jones, B., Weaving, D., Tee, J., Darrall-Jones, J., Weakley, J., Phibbs, P., Read, D., Roe, G., Hendricks, S. \& Till, K., Bigger, stronger, faster, fitter: the differences in physical qualities of school and academy rugby union players, Journal of Sports Sciences, 2018, 36(21), 2399-2404. 
11. Bertollo M, Berchicci M, Carraro A, Comani S, Robazza C., Blocked and random practice organization in the learning of rhythmic dance step sequences, Perceptual and Motor Skills, 2010, 110(1), 77-84.

12. Angioi $M$, Metsios $G$, Koutedakis $Y$, Wyon MA., Fitness in contemporary dance: a systematic review, International Journal of Sports Medicine, 2009, 30(07), 475-84.

13. Bompa, T and Buzzichelli, C., Periodization: Theory and Methodology of training. $6^{\text {th }}$ ed. Champaign, IL: Human Kinetics, 2018, p.93.

14. Haff, G.G., 17 The essentials of periodisation, Strength and Conditioning for Sports Performance, 2016,404.

15. Tee JC, Ashford M, Piggott D., A tactical periodization approach for rugby union, Strength and Conditioning Journal, 2018, 40(5), 1-3.

16. Koutedakis, Y., Clarke, F., Wyon, M., Aways, D. \& Owolabi, E.O., Muscular strength: Applications for dancers, Medical Problems of Performing Artists, 2009, 24(4), 157-65.

17. Mistiaen W, Roussel NA, Vissers D, Daenen L, Truijen S, Nijs J., Effects of aerobic endurance, muscle strength, and motor control exercise on physical fitness and musculoskeletal injury rate in preprofessional dancers: an uncontrolled trial, Journal of Manipulative and Physiological Therapeutics, 2012, 35(5), 381-9.

18. Alpert PT., The health benefits of dance, Home Health Care Management and Practice, 2011, 23(2), 155-7.

19. Malkogeorgos A, Zaggelidou E, Georgescu L., The Effect of Dance Practice on Health, Asian Journal of Exercise and Sports Science, 2011, 8(1).

20. Kiepe MS, Stöckigt B, Keil T., Effects of dance therapy and ballroom dances on physical and mental illnesses: A systematic review, The Arts in Psychotherapy, 2012, 39(5), 404-11.

21. Murrock CJ, Graor $\mathrm{CH}$., Effects of dance on depression, physical function, and disability in underserved adults, Journal of Aging and Physical Activity, 2014, 22(3), 380-5.

22. Sharp K, Hewitt J., Dance as an intervention for people with Parkinson's disease: a systematic review and meta-analysis, Neuroscience and Biobehavioral Reviews, 2014, 47, 445-56. 
23. Iftekher SN, Bakhtiar M, Rahaman KS., Effects of yoga on flexibility and balance: a quasi-experimental study, Asian Journal of Medical and Biological Research, 2017, 3(2), 276-81.

24. Polsgrove MJ, Eggleston BM, Lockyer RJ., Impact of 10-weeks of yoga practice on flexibility and balance of college athletes, International Journal of Yoga, 2016, 9(1), 27.

25. Koutedakis, Y., \& Jamurtas, A., The dancer as a performing athlete. Sports medicine, 2004, 34(10), 651-661.

26. Vaz L, Abade E, Fernandes MH, Reis MV., Cross-training in rugby: A review of research and practical suggestions, International Journal of Performance Analysis in Sport, 2013, 13(1), 225-37.

27. Jarvis, S., Sullivan, L.O., Davies, B., Wiltshire, H. and Baker, J.S., Interrelationships between measured running intensities and agility performance in subelite rugby union players. Research in Sports Medicine, 2009, 17(4), pp.217230.

28. Cahill, N., Lamb, K., Worsfold, P., Headey, R. and Murray, S., The movement characteristics of English Premiership rugby union players. Journal of Sports Sciences, 2013, 31(3), pp.229-237.

29. Meir, R., Newton, R., Curtis, E., Fardell, M. and Butler, B., Physical fitness qualities of professional rugby league football players: determination of positional differences. The Journal of Strength \& Conditioning Research, 2001, 15(4), pp.450-458.

30. Calmeyer, T., De Kok, C., Hardy, J., Rogers, J.A. \& Taljaard, L., How to succeed in your master's and doctoral studies: A South African guide and resource book, Johann Mouton book review, New Voices in Psychology, 2001, 7(2), 148-152.

31. Dwan K, Li T, Altman DG, Elbourne D., CONSORT 2010 statement: extension to randomised crossover trials, British Medical Journal, 2019, 366, 14378.

32. Clarke, A., \& Dawson, R., Understanding evaluation. Evaluation Research: an Introduction to Principles, Methods and Practice, 1999, 1, 34.

33. Senn, SJ., Base logic: tests of baseline balance in randomized clinical trials. Clinical Research and Regulatory Affairs, 1995, 12(3), 171-82.

34. Hecksteden A, Faude O, Meyer T, Donath L., How to construct, conduct and analyze an exercise training study?, Frontiers in Physiology, 2018, 9. 
35. South African Rugby, Pre-season Testing and the Physical Profiling of Players, 20 June 2020: https://www.springboks.rugby/en/pages/BokSmart-MedicalProtocol-Profiling-of-Players.

36. Mayorga-Vega, D., Merino-Marban, R. and Viciana, J., Criterion-related validity of sit-and-reach tests for estimating hamstring and lumbar extensibility: A metaanalysis. Journal of Sports Science \& Medicine, 2014, 13(1), p.1.

37. Gribble, P.A., Hertel, J. and Plisky, P., Using the Star Excursion Balance Test to assess dynamic postural-control deficits and outcomes in lower extremity injury: a literature and systematic review, Journal of Athletic Training, 2012. 47(3), pp.339-357.

38. Moran, M.K., Wagganer, J.D., Jones, E.J., Bergman, R.J. and Pujol, T.J., Validation of a One-Minute Abdominal, 2018.

39. Räisänen, A., Pasanen, K., Krosshaug, T., Avela, J., Perttunen, J. and Parkkari, J., Single-Leg squat as a tool to evaluate young athletes' frontal plane knee control, Clinical Journal of Sport Medicine, 2016, 26(6), pp.478-482.

40. Barr MJ, Sheppard JM, Agar-Newman DJ, Newton RU., Transfer effect of strength and power training to the sprinting kinematics of international rugby players, Journal of Strength and Conditioning Research, 2014, 28(9), 2585-96.

41. Kloubec JA., Pilates for improvement of muscle endurance, flexibility, balance, and posture, Journal of Strength and Conditioning Research, 2010, 24(3), 6617.

42. Duthie G, Pyne D, Hooper S., Applied physiology and game analysis of rugby union, Journal of Sports Medicine, 2003, 33(13), 973-91.

43. Durandt J, Du Toit S, Borresen J, Hew-Butler T, Masimla H, Jokoet I, Lambert M., Fitness and body composition profiling of elite junior South African rugby players, South African Journal of Sports Medicine, 2006, 18(2), 38-45.

44. Quarrie KL, Hopkins WG, Anthony MJ, Gill ND., Positional demands of international rugby union: Evaluation of player actions and movements, Journal of Science and Medicine in Sport, 2013, 16(4), 353-9.

45. Côté-Laurence, P., The role of rhythm in ballet training, Research in Dance Education, 2000, 1(2), 173-91. 